University of Nebraska - Lincoln

DigitalCommons@University of Nebraska - Lincoln

Faculty Publications: Materials Research

Science and Engineering Center

Materials Research Science and Engineering

Center

2006

\title{
Effects of ion-beam irradiation on the L10 phase transformation and their magnetic properties of FePt and PtMn films (Invited)
}

Chih-Huang Lai

National Tsing Hua University, 300, HsinChu, Taiwan

Sheng-Huang Huang

National Tsing Hua University, 300, HsinChu, Taiwan

Cheng-Han Yang

National Tsing Hua University, 300, HsinChu, Taiwan

C.C. Chiang

National Tsing Hua University, 300, HsinChu, Taiwan

Sy_Hwang Liou

University of Nebraska-Lincoln, sliou@unl.edu

See next page for additional authors

Follow this and additional works at: https://digitalcommons.unl.edu/mrsecfacpubs

Part of the Materials Science and Engineering Commons

Lai, Chih-Huang; Huang, Sheng-Huang; Yang, Cheng-Han; Chiang, C.C.; Liou, Sy_Hwang; Sellmyer, David J.; Yan, M. L.; Yuan, L.; and Yokata, T., "Effects of ion-beam irradiation on the L10 phase transformation and their magnetic properties of FePt and PtMn films (Invited)" (2006). Faculty Publications: Materials Research Science and Engineering Center. 78.

https://digitalcommons.unl.edu/mrsecfacpubs/78

This Article is brought to you for free and open access by the Materials Research Science and Engineering Center at DigitalCommons@University of Nebraska - Lincoln. It has been accepted for inclusion in Faculty Publications:

Materials Research Science and Engineering Center by an authorized administrator of DigitalCommons@University of Nebraska - Lincoln. 


\section{Authors}

Chih-Huang Lai, Sheng-Huang Huang, Cheng-Han Yang, C.C. Chiang, Sy_Hwang Liou, David J. Sellmyer, M. L. Yan, L. Yuan, and T. Yokata 


\title{
Effects of ion-beam irradiation on the $\mathrm{L1}_{0}$ phase transformation and their magnetic properties of FePt and PtMn films (Invited)
}

Chih-Huang Lai ${ }^{1}$, Sheng-Huang Huang ${ }^{1}$, Cheng-Han Yang ${ }^{1}$, C.C. Chiang ${ }^{1}$, S. H. Liou ${ }^{2}$, D. J.

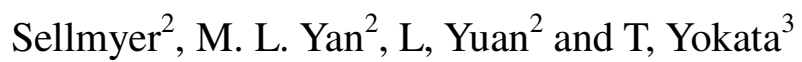

${ }^{1}$ Department of Materials Science and Engineering, National Tsing Hua University, 300, HsinChu, Taiwan.

${ }^{2}$ Department of Physics and Astronomy, University of Nebraska, Lincoln, NE 68588

${ }^{3}$ Department of Environmental Engineering of Materials, Nagoya institute of Technology, Nagoya, Japan.

\begin{abstract}
In this paper, we illustrate how to modify the structure and magnetic properties of $\mathrm{L}_{0} \mathrm{FePt}$ and PtMn films using ion-beam irradiation. Highly ordered $\mathrm{L}_{0}$ FePt and PtMn phases were achieved directly by using $2 \mathrm{MeV}$ He-ion irradiation without conventional post-annealing. A high ion-beam current density $\left(\sim \mu \mathrm{A} / \mathrm{cm}^{2}\right)$ was used to achieve direct beam heating on samples. This irradiation-induced heating process provides efficient microscopic energy transfer and creates excess point defects, which significantly enhances the diffusion and promotes the formation of the ordered $\mathrm{L}_{0}$ phase. In-plane coercivity of FePt films greater than $5700 \mathrm{Oe}$ could be obtained after disordered FePt films were irradiated with a He-ion dose of $2.4 \times 10^{16}$ ions $/ \mathrm{cm}^{2}$. The direct ordering of FePt took place by using ion-irradiation heating at a temperature as low as $230{ }^{\circ} \mathrm{C}$. In PtMn-based spin valves, an $\mathrm{L} 1_{0} \mathrm{PtMn}$ phase, a large exchange field and a high giant magnetoresistance (GMR) ratio (11\%) were simultaneously obtained by using He-ion irradiation. On the other hand, Ge-ion and O-ion irradiation completely destroyed the ferromagnetism of FePt and the GMR of PtMn-based spin valves, respectively.
\end{abstract}

\section{INTRODUCTION}

To develop a high-density magnetic recording system, sensitive reading elements and high-coercivity media are essential. Currently, the readers are composed of a spin-valve structure in which an antiferromagnetic (AFM) layer provides a strong exchange bias to pin the adjacent ferromagnetic (FM) layer. A typical material used for the AFM layer is PtMn. The applications of exchange anisotropy stimulated extensive theoretical and experimental studies [1-2]. It has been found when increasing areal density of media, the super-paramagnetic limit becomes a critical issue, especially when the grain size is reduced to below $5 \mathrm{~nm}$. L1 $1_{0} \mathrm{FePt}$ 
possesses high magnetocrystalline anisotropy $\left(\mathrm{K}_{\mathrm{u}} \sim 7 \times 10^{7} \mathrm{erg} / \mathrm{cc}\right)$ [3] and is one of most promising candidates for ultra-high-density media. Both as-deposited PtMn and FePt are disordered fcc phases, and post-annealing is needed to transform the disordered to ordered $\mathrm{L}_{0}$ phase. The typical annealing temperature for PtMn is around 250 to $280{ }^{\circ} \mathrm{C}$, and that for FePt is higher than $500{ }^{\circ} \mathrm{C}$.

When magnetic recording systems forge ahead to a small scale, it is important, therefore, to develop novel processing methods. In recent years, ion irradiation has drawn much attention as a potential tool to modify the magnetic properties without affecting the surface topography. For example, changing the anisotropy of $(\mathrm{Co} / \mathrm{Pt})$ multilayers [4], varying the GMR ratio of $(\mathrm{Fe} / \mathrm{Cr})$ multilayers [5] and enhancing the magnetic moment of CoPt alloys [6] were reported. In this work, we investigated the ion-irradiation effects on the magnetic thin films composed of $\mathrm{L}_{0}$ phases. Two topics are discussed: (A) the FePt films and (B) the spin-valve structure with PtMn layers.

Typically, as-deposited FePt films of disordered fcc phase are magnetically soft. Post-annealing at temperatures higher than $500^{\circ} \mathrm{C}$ is needed to develop an ordered $\mathrm{L} 1_{0}$ phase and to achieve a large coercivity. He-ion irradiation was used to control the degree of chemical ordering of FePt films [7]. The long range ordering factor S of sputtered FePt (001) films can be improved by using post-growth irradiation. But only a film which already has a partially ordered structure $(\mathrm{S}=0.4)$ can develop an induced long-range ordered phase. Ion irradiation can introduce an appropriate amount of atomic species and desired energy into the films. The energetic incident ions may create defects or lattice distortion and thus may promote phase transformation. In part (A) of the results and discussion, we report that the ordering of $\mathrm{FePt} \mathrm{L}_{0}$ phase can be directly induced by $2 \mathrm{MeV}$ He-ion irradiation. We purposely adjusted the beam current to a $\mu \mathrm{A} / \mathrm{cm}^{2}$ scale to study the beam heating effect and the energy transfer in FePt films. In addition, the comparisons were made between rapid thermal annealing (RTA) and ion irradiation. We also propose a method of magnetic patterning for patterned media by using ion irradiation.

Ion irradiation with lithographic masks has been proposed as an alternative technique for making patterned magnetic nanostructures, and for defining the widths of tracks in giant magnetoresistance (GMR) sensors [8]. In the case of ferromagnetic/antiferromagnetic (FM/AFM) exchange-biased bilayers, the exchange bias is sensitive to the interface and the AFM anisotropic energy. Accordingly, ion irradiation has been used to change both the magnitude and direction of the exchange field [9]. The exchange field can be either enhanced by the creation of defects, acting as domain-wall pinning sites, or can be suppressed to zero by the intermixing. C-ion irradiation has been applied to modify the exchange field and GMR ratios of PtMn- and NiMn- based spin valves. C-ion irradiation increases the exchange field because 
of the formation of $\operatorname{PtMn}(\mathrm{C})$ and $\mathrm{NiMn}(\mathrm{C})$ phases whose c/a ratio considerably less than that of the parent phases [10-11]. However, the GMR ratio decreased because of the C-residuals at the $\mathrm{CoFe} / \mathrm{Cu}$ interfaces. In part $(\mathrm{B})$ of the results and discussion, we demonstrate that a high exchange field and GMR ratio of the PtMn-based spin valves can be achieved by using $2 \mathrm{MeV}$ He-ion without post-annealing. Furthermore, we propose a method of magnetic patterning to define the dimension of spin-valve sensors by using ion irradiation.

\section{EXPERIMENTAL PROCEDURE}

In part (A), $50 \mathrm{~nm} \mathrm{FePt} \mathrm{films} \mathrm{were} \mathrm{deposited} \mathrm{on} \mathrm{SiO}_{2} / \mathrm{Si}$ substrates at room temperature by using co-sputtering from elemental targets. The composition of the FePt films is $\mathrm{Fe}_{0.5} \mathrm{Pt}_{0.5}$, measured by Rutherford Backscattering Spectrometry (RBS). The He-ion irradiation was performed on as-deposited disordered FePt films at ambient temperature with an energy of 2 $\mathrm{MeV}$ by using a KN accelerator. The beam current was set between $1.25 \mu \mathrm{A} / \mathrm{cm}^{2}$ and $6 \mu \mathrm{A} / \mathrm{cm}^{2}$. The temperature on the sample surface during irradiation was in-situ monitored by an attached thermocouple. Computer simulation SRIM code [12] enabled us to predict the depth profile of He ions, the energy loss, and the defect creation in the FePt films. The hysteresis loops were measured by using a vibrating sample magnetometer (VSM). X-ray diffraction (XRD) $\theta-2 \theta$ scans using the $\mathrm{Cu} \mathrm{K} \mathrm{K}_{\alpha}$ line provided the structural identification. The ordering factor $\mathrm{S}$ was directly deduced from the c/a ratio [13].

In part (B), the structure of spin-valve samples is $\mathrm{Si} / / \mathrm{NiFeCr} 5 / \mathrm{NiFe} 3 / \mathrm{CoFe} 1.5 / \mathrm{Cu} 2.6 /$ CoFe 2.2/ PtMn 20/ NiFeCr 5 (unit: nm). The bottom and top NiFeCr layers are employed to improve the (111) texture and prevent oxidation, respectively. The composition of PtMn is $\mathrm{Pt}_{50} \mathrm{Mn}_{50}$. The as-deposited PtMn is a disordered fcc phase. The PtMn-based spin valves are irradiated with He-ion at ambient temperature using a $\mathrm{KN}$ accelerator. The beam current is 1.08 $\mu \mathrm{A} / \mathrm{cm}^{2}$, with a corresponding temperature at the sample surface about $190{ }^{\circ} \mathrm{C}$, as measured using an in situ thermocouple, which was placed directly on the sample surface during irradiation. The He-ion dose was varied from $6.96 \times 10^{15}$ to $1.74 \times 10^{16}$ ions $/ \mathrm{cm}^{2}$. An external field of 900 Oe was applied during irradiation to ensure the unidirectional anisotropy of PtMn. The acceleration voltage was $2 \mathrm{MeV}$; the calculated projected range $(\mathrm{Rp})$ is about $7.22 \mu \mathrm{m}$ and the straggle range $(\Delta \mathrm{Rp})$ is about $0.227 \mu \mathrm{m}$, according to the SRIM simulation program. Hence, the majority of He-ions were implanted into the silicon substrates. On the other hand, $42 \mathrm{keV}$ O-ion irradiation was performed using a NEC 9SDH2 accelerator following the irradiation of the spin-valve samples with $2 \mathrm{MeV}$ He-ions. The hysteresis loops were measured using a vibrating sample magnetometer (VSM). X-ray diffraction (XRD) $\theta-2 \theta$ scans using the $\mathrm{Cu} \mathrm{K}_{\alpha}$-line 
elucidated the film structure. The GMR ratio was measured using a four-point probe at room temperature.

\section{RESULTS AND DISCUSSION}

\section{Part (A). Ion-irradiation effects on FePt films}

The hysteresis loop of the FePt film irradiated at the beam current of $1.25 \mu \mathrm{A} / \mathrm{cm}^{2}$ with an ion dose of $2.4 \times 10^{16}$ ions $/ \mathrm{cm}^{2}$ is shown in Fig. 1. The coercivity of the irradiated sample is as high as 5700 Oe. The temperature on the surface of the irradiated FePt film during irradiation was about $230{ }^{\circ} \mathrm{C}$ due to direct beam heating. As shown in Fig. 2, XRD scans reveal the evolution of the structural ordering in the irradiated FePt film. The (001) superlattice peak was observed after irradiation. Also, the (111) fundamental peak of the irradiated FePt film shifted toward the higher angle side, which indicates that the fcc disordered phase has been partially transformed into the $\mathrm{L1}_{0}$ ordered phase. For comparison, we annealed an unirradiated sample using a conventional vacuum furnace at $230{ }^{\circ} \mathrm{C}$ for $10 \mathrm{~h}$. The ordered peaks of FePt after conventional furnace annealing is unapparent, as shown in Fig. 2, leading to a low coercivity of 1200 Oe (as shown in Fig. 1).

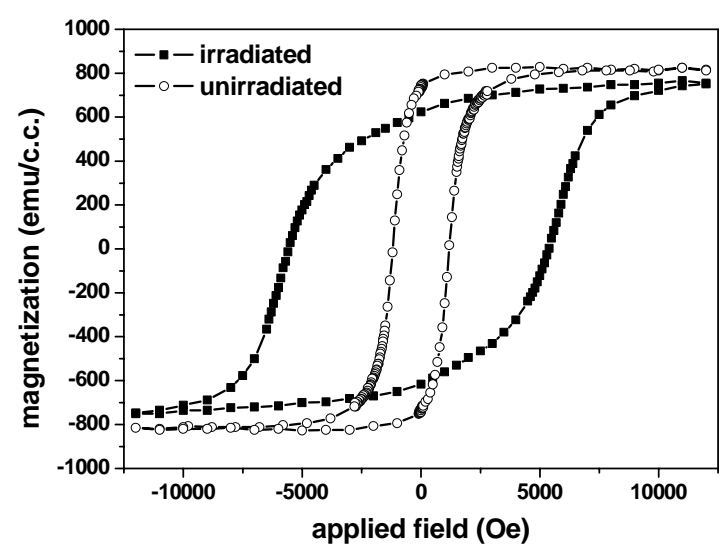

Figure 1. Hysteresis loops of irradiated and unirradiated FePt films. The He-irradiation was performed at a beam current of $1.25 \mu \mathrm{A} / \mathrm{cm}^{2}$ with an ion dose of $2.4 \times 10^{16}$ ions $/ \mathrm{cm}^{2}$. The unirradiated FePt film was annealed in a furnace at $230{ }^{\circ} \mathrm{C}$ for $10 \mathrm{~h}$. 


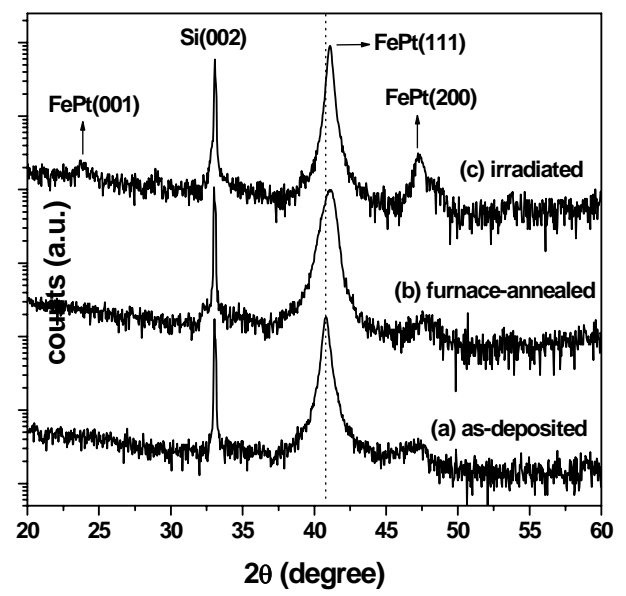

Figure 2. X-ray $\theta-2 \theta$ scans of (a) the as-deposited FePt film, (b) the unirradiated FePt film annealed in a vacuum furnace at $230{ }^{\circ} \mathrm{C}$ for $10 \mathrm{~h}$, (c) the FePt film irradiated at a beam current of $1.25 \mu \mathrm{A} / \mathrm{cm}^{2}$ with a dose of $2.4 \times 10^{16}$ ions $/ \mathrm{cm}^{2}$. (The dash line is used to indicate peak shift).

The conventional post-annealing at $230{ }^{\circ} \mathrm{C}$ was not sufficient to provide enough energy for the ordering of FePt films. Instead, by using an ion-irradiation process, the energy transfer from He-ions to FePt films as well as point defects and lattice distortion induced by irradiation led to direct ordering. Conventional annealing only provides atoms extra thermal energy to overcome the activation energy of phase transformation. In contrast, the ion irradiation with a high beam-current-density not only provides beam heating but energy transfer from incident ions. The energy dissipation may cause the temperature to rise significantly within several nanometers around the moving ions and then rapidly quench to ambient temperature [14]. The atoms in the irradiated FePt films, therefore, have been relaxed from the original lattice points due to a succession of particle collisions and interaction. Combining the relaxation of the ion-disturbed FePt atoms by energy transfer with the beam heating to $230{ }^{\circ} \mathrm{C}$, the mobility of the FePt atoms has been enhanced considerably.

During the ordering process, the diffusivity of $\mathrm{Fe}$ and $\mathrm{Pt}$ atoms in FePt films determines the transformation rate and the ordering factor. According to SRIM code simulation, the defect concentration created in the irradiated FePt film by an ion dose of $2.4 \times 10^{16}$ ions $/ \mathrm{cm}^{2}$ is $7.15 \times 10^{21}$ defects $/ \mathrm{cm}^{3}$ which is still much lower than the atom density of FePt. That is, FePt can still maintain its lattice without severe distortion. On the other hand, excess point defects such as vacancies and interstitials under irradiation are formed with a much higher concentration than that created by conventional annealing. The excess point defects can greatly increase the diffusivity of the Fe and $\mathrm{Pt}$ atoms. Consequently, irradiation-enhanced diffusion promotes the 
ordering of irradiated FePt films. Moreover, the sample surface is directly heated up by the ion beam, which further increases diffusivity. The irradiation-induced thermal heating process, providing a microscopic energy exchange and enhanced diffusion, is more efficient than any other conventional annealing process. As a result, a highly ordered $\mathrm{L1}_{0}$ phase $(\mathrm{S}=0.73)$ of the FePt film was obtained with irradiation-beam heating at a low temperature of $230{ }^{\circ} \mathrm{C}$.

Fig. 3 illustrates the dependence of the coercivity $\mathrm{H}_{\mathrm{c}}$ and ordering factor $\mathrm{S}$ on beam current for the samples irradiated at the same ion dose of $2.4 \times 10^{16}$ ions $/ \mathrm{cm}^{2}$. By increasing beam current, the temperature on the sample surface was raised from $230{ }^{\circ} \mathrm{C}$ to $600^{\circ} \mathrm{C}$. This is consistent with the $\mathrm{W}$-ion irradiation in $\mathrm{Si}$ where the temperature of the ion flux is proportional to the beam current [15]. By increasing beam current, the beam heating effect is more pronounced and enhances the diffusivity so more volume fractions of ordered $\mathrm{L}_{0}$ phase were formed, leading to increased $\mathrm{H}_{\mathrm{c}}$ and $\mathrm{S}$.

Based on the previous results, we believe that the direct ordering of FePt can be obtained at the nominal temperature as low as $230{ }^{\circ} \mathrm{C}$ by using irradiation-heating process. This process involves several effects including microscopic energy transfer, excess point defects, and direct beam heating. We designed sets of experiments to understand which effects really affect the ordering of FePt. Porous anodic aluminum oxides (AAOs) were used as masks during irradiation. The AAO substrate with a thickness of $60 \mu \mathrm{m}$ and a hole size of $100 \mathrm{~nm}$ on average is viewed as a beehive-like structure. $2 \mathrm{MeV}$ He-ion irradiation with a beam current of $1.25 \mu \mathrm{A} / \mathrm{cm}^{2}$ and an ion dose of $2.4 \times 10^{16}$ ions $/ \mathrm{cm}^{2}$ was performed on the $\mathrm{FePt} / \mathrm{SiO}_{2} / \mathrm{Si}$ sample with a porous AAO mask. The surface temperature of irradiated sample during irradiation was also $230^{\circ} \mathrm{C}$.

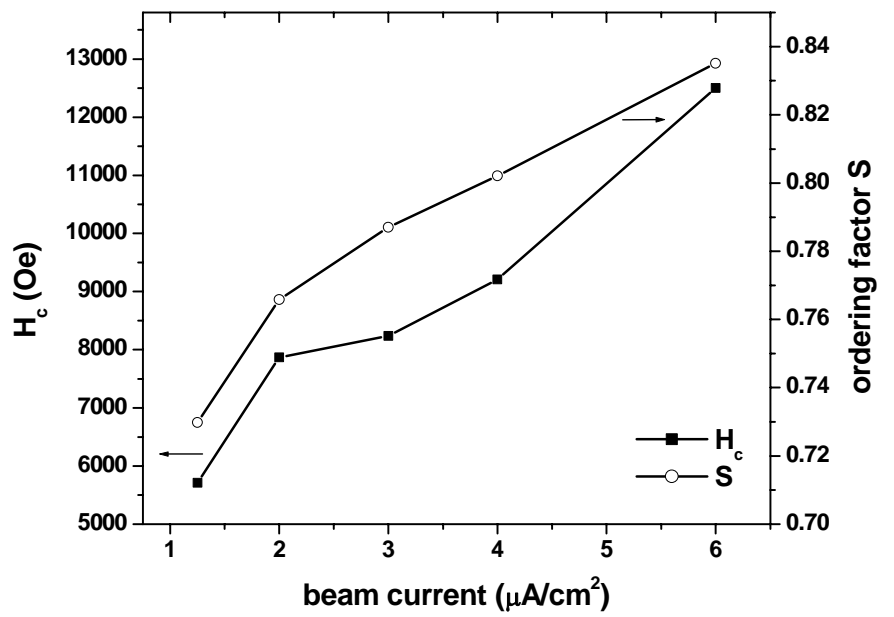

Figure 3. Dependence of coercivity $\mathrm{H}_{c}$ and ordering factor $\mathrm{S}$ on beam current for the samples irradiated at an ion dose of $2.4 \times 10^{16}$ ions $/ \mathrm{cm}^{2}$. 
The hysteresis loop of the irradiated sample with an AAO mask is identical to that of as-deposited samples. Since the aspect ratio (width to depth ratio) of the holes of porous AAO is quite large, only extremely straight and collimated incident He-ions can go through the porous AAO into FePt films. As a result, most He ions stopped in the AAO and the ordered FePt $\mathrm{L}_{0}$ phase cannot be obtained. In other words, although the ion beam directly heated up the sample, the microscopic energy transfer between incident ions and FePt atoms must be still required to achieve the ordering of FePt films. In addition, we performed Ni-ion irradiation with an energy of $8.8 \mathrm{MeV}$ and a dose of $2.4 \times 10^{16}$ ions $/ \mathrm{cm}^{2}$ on the $\mathrm{FePt} / \mathrm{SiO}_{2} / \mathrm{Si}$ films. The beam current was kept at $300 \mathrm{nA} / \mathrm{cm}^{2}$. The temperature of the irradiated sample surface monitored by the thermocouple is $40{ }^{\circ} \mathrm{C}$. Notice that all $\mathrm{Ni}$ ions penetrated through the film into the substrates according to the simulation of SRIM code. The energy loss in the FePt layer via electronic stopping was $800 \mathrm{eV} / \AA$ and the defect concentration was $2.25 \times 10^{24}$ defects $/ \mathrm{cm}^{3}$, both of which are much higher than those of $2 \mathrm{MeV}$ He-ion irradiation. The hysteresis loop of as-irradiated $\mathrm{FePt}$ films shows reduced $\mathrm{H}_{\mathrm{c}}$ compared to as-deposited films. Although Ni-irradiation provides high energy transfer and excess point defects, FePt films still require direct beam heating to accelerate the diffusion and phase transformation. The reduced $\mathrm{H}_{\mathrm{c}}$ could be due to reduction in domain wall energy by ion bombardment. Based on these results, all three effects: energy transfer, excess point defects and direct beam heating are essential for low-temperature ordering of $\mathrm{L}_{0}$ FePt by using ion irradiation.

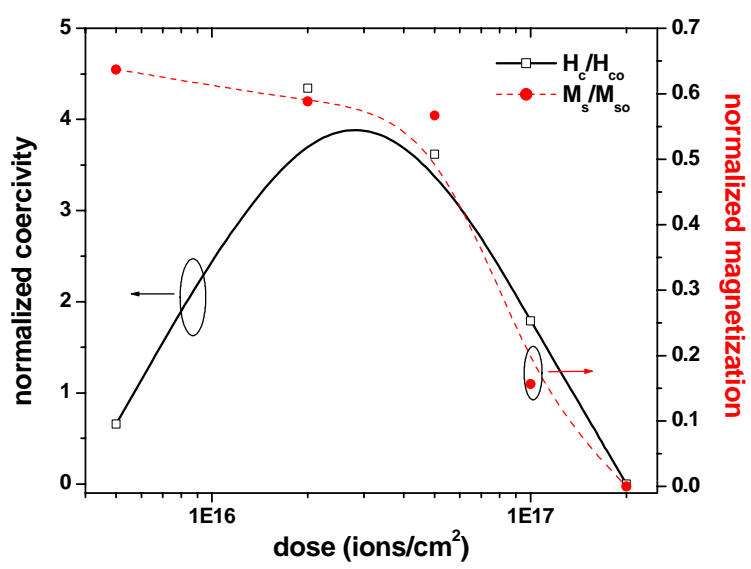

Figure 4. Dependence of normalized coercivity and magnetization on the Ge ion dose. Both values are normalized by the as-deposited disordered FePt values.

By using He-ion irradiation, a high coercivity of FePt can be obtained after irradiation. On the other hand, Ge-ion irradiation shows completely opposite effects. $75 \mathrm{keV} \mathrm{Ge}$-ion irradiation 
was performed on the as-deposited disordered FePt films. The beam current was fixed at 300 $\mathrm{nA} / \mathrm{cm}^{2}$. The accelerating voltage was chosen to let a majority of Ge remain in the FePt films. The coercivity and saturation magnetization of the irradiated samples normalized by the values of as-deposited disordered films are shown in Fig. 4. When the dose was increased to $2 \times 10^{17}$ ions $/ \mathrm{cm}^{2}$, the coercivity and magnetization approached zero. Consequently, Ge-ion irradiation destroyed the ferromagnetism of FePt. The irradiated Ge atoms may dilute the Fe concentration and reduce the Curie temperature to below room temperature.

Due to distinctively different results on the coercivity of FePt by using $\mathrm{Ge}$ and $\mathrm{He}$ ion irradiation, a novel approach of magnetic patterning is proposed to fabricate patterned media. The schematic diagram for this approach is illustrated in Fig. 5. A patterned FePt film was covered by resist, which has patterned metals on the top. The thickness and kind of metals are determined by the irradiation parameters and decide the depth profile of irradiated species in FePt films. The first step is to irradiate Ge-ions into the uncovered area (step (a)). The ferromagnetism of the irradiated area is destroyed by Ge-ion irradiation. The resist and metals are subsequently removed (step (b)). In step (c), He-ion irradiation is performed on the whole wafer. After irradiation, the wafer is divided into two regions, showing different magnetic signals during reading. The region, originally covered by metal and resist in step (a) during Ge-ion irradiation, shows a high coercivity and a strong magnetic signal because the $\mathrm{L} 1_{0} \mathrm{FePt}$ phase was directly induced by He-ions in step (c). The other region, which is uncovered and exposed to Ge-ion irradiation in step (a), shows no magnetic signal. Consequently, the patterned media can be achieved by using ion irradiation without physical etching.

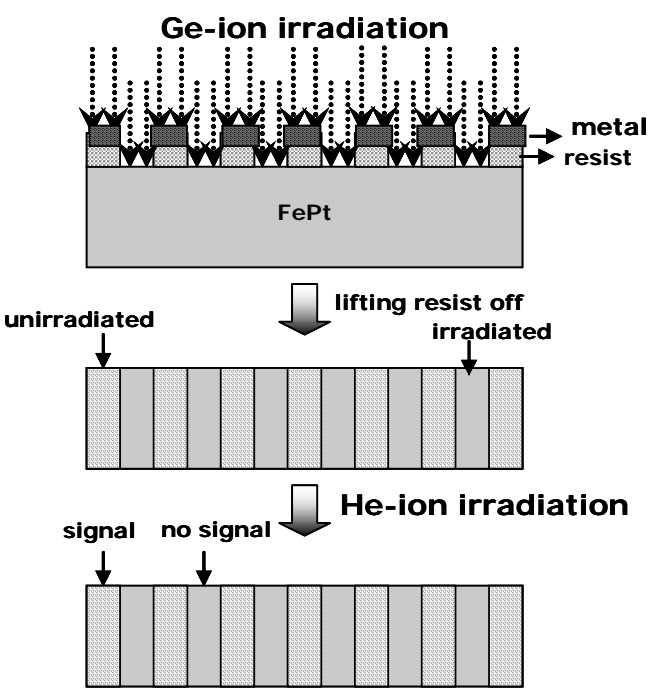

(b)

(c)

Figure 5. Schematic diagram of fabrication for patterned media by using ion irradiation. (a) Ge-ion irradiation is first performed, (b) resist and metals are removed and (c) He-ion irradiation is performed. The areas exposed to Ge-ion irradiation show no magnetic signal, but the rest 
area shows a high coercity and magnetic signal.

Comparisons of rapid thermal annealing (RTA) and ion-irradiation were carried out on the $[\mathrm{Fe}(4.8 \AA) / \operatorname{Pt}(5 \AA)]_{10} / \mathrm{C}(100 \AA)$ films. Typical in-plane and out-plane magnetization curves for the sample annealed at $550{ }^{\circ} \mathrm{C}$ for 300 secs by RTA method are shown in Fig 6 (a). The sample annealed at $550{ }^{\circ} \mathrm{C}$ by RTA has perpendicular anisotropy with a coercivity of $6 \mathrm{kOe}$. It also showed (001) texture in XRD spectra. The $2 \mathrm{MeV}$ He-ion irradiated sample with a current-density of $5.04 \mu \mathrm{A} / \mathrm{cm}^{2}$ for $360 \mathrm{secs}$, which corresponds to a surface temperature of 550 ${ }^{\circ} \mathrm{C}$, has a coercivity as large as $10 \mathrm{kOe}$ but no significant difference in the in-plane and out-of plane coercivity, as shown in Fig. 6 (b). All ion-irradiated samples have (111) textures, which lead to isotropic magnetic properties. These results may suggest that the structural evolution during the RTA and ion-irradiation process may be quite different, resulting in different textures. The structural modification by ion irradiation needs to be taken into account when ion irradiation is used for magnetic patterning.

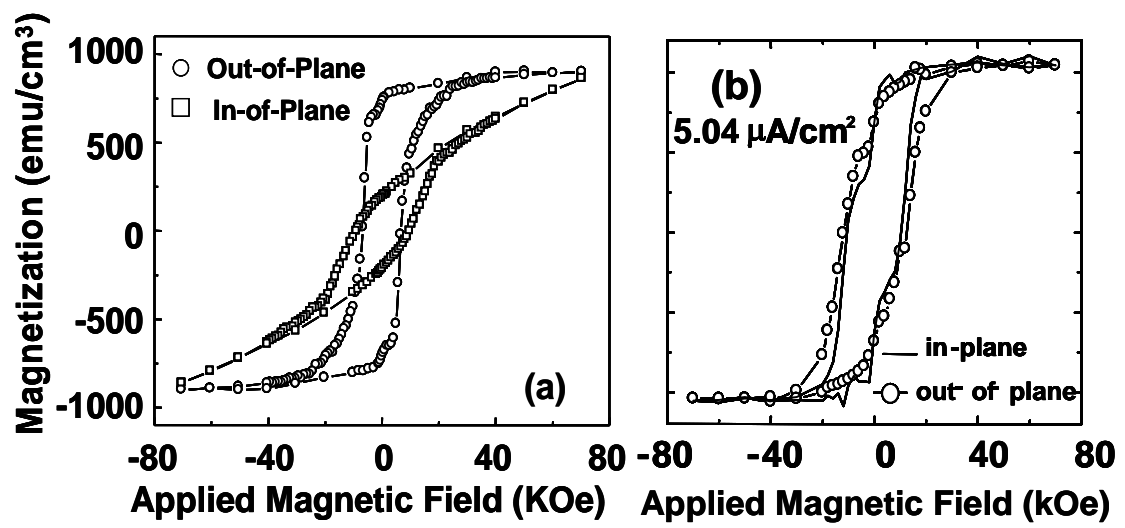

Figure 6. Magnetization curves for the sample (a) annealed at $550{ }^{\circ} \mathrm{C}$ for $300 \mathrm{sec}$ by RTA method and (b) irradiated by using $2 \mathrm{MeV}$ He-ions with current density of $5.04 \mu \mathrm{A} / \mathrm{cm}^{2}$ for 360 secs

\section{Part (B). Ion-irradiation effects on the spin-valve structure with PtMn layers}

Figs. 7(a) and 8(a) show the hysteresis and MR loops, respectively, after He-irradiation. The irradiation dose is $1.91 \times 10^{16}$ ions $/ \mathrm{cm}^{2}$. The exchange field and the MR ratio after irradiation are 650 Oe and 11\%, respectively. For comparison, Figs. 7(b) and 8(b) present the hysteresis and MR loops of as-deposited samples, respectively. The exchange field and MR appeared after irradiation because of formation of the ordered PtMn phase, which was verified by the shift of the PtMn (111) peak in X-ray diffraction patterns. The MR ratio strongly 
depends on the interfacial conditions of $\mathrm{CoFe} / \mathrm{Cu} / \mathrm{CoFe}$. The $\mathrm{MR}$ ratio of $11 \%$ is almost the same as that obtained by furnace annealing at $280{ }^{\circ} \mathrm{C}$ for 2 hours, which may demonstrate that He-ion irradiation does not induce severe interfacial changes because $\mathrm{Rp}$ is large. Similar to $\mathrm{N}$-ion irradiation for current-in-plane (CIP) GMR pattering [8], He-ion irradiation can be used for defining sizes of CIP sensors composed of PtMn-based spin valves. The irradiated area shows a high GMR ratio, but non-irradiated area shows almost zero GMR. The schematic diagram of magnetic patterning for spin valve sensors is shown in Fig. 9 (a). Furthermore, the direction of unidirectional anisotropy can be controlled by changing the direction of the external field during irradiation. Consequently, ion irradiation combined with a lithographic mask can enable us to fabricate GMR cells with different pining directions on the same wafer. This process outperforms the conventional post-annealing process, which can only give single pinning direction on one wafer by an external field.

To eliminate MR and reduce the current shunting effect in the irradiated area, $42 \mathrm{keV}$ O-ion irradiation was used to fabricate patterned GMR. First, GMR samples were irradiated with He ions at a fluence of $1.09 \times 10^{16}$ ions $/ \mathrm{cm}^{2}$. An $11 \% \mathrm{MR}$ ratio was achieved and O-ion irradiation was again applied. The MR ratio dropped and approached zero following O-ion irradiation at a fluence of $2 \times 10^{16} \mathrm{ions} / \mathrm{cm}^{2}$. Another approach for magnetic patterning for spin valve sensors is schematically shown in Fig. 9 (b).

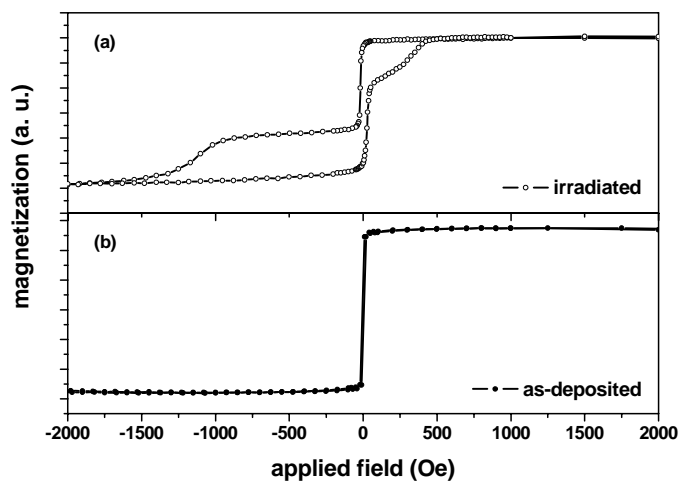

Figure 7. Hysteresis loops of (a) irradiated and (b) as-deposited spin valves. The irradiation fluence is $1.91 \times 10^{16}$ ions $/ \mathrm{cm}^{2}$.

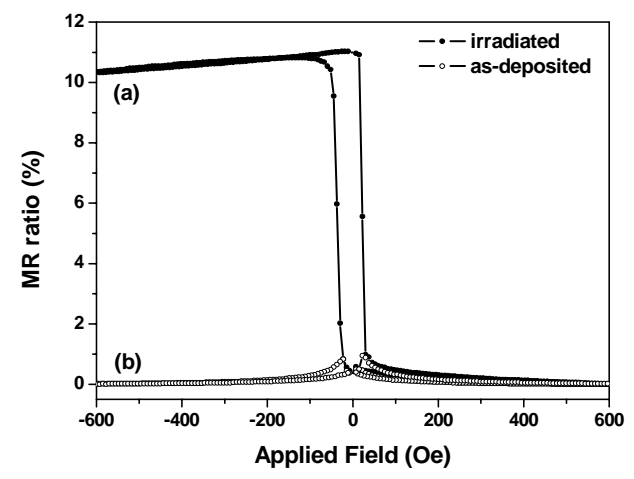

Figure 8. MR curves of (a) irradiated and (b) as-deposited spin valve samples. The irradiation fluence is $1.91 \times 10^{16}$ ions $/ \mathrm{cm}^{2}$. 
(a) $2 \mathrm{MeV}$ He-ion irradiation

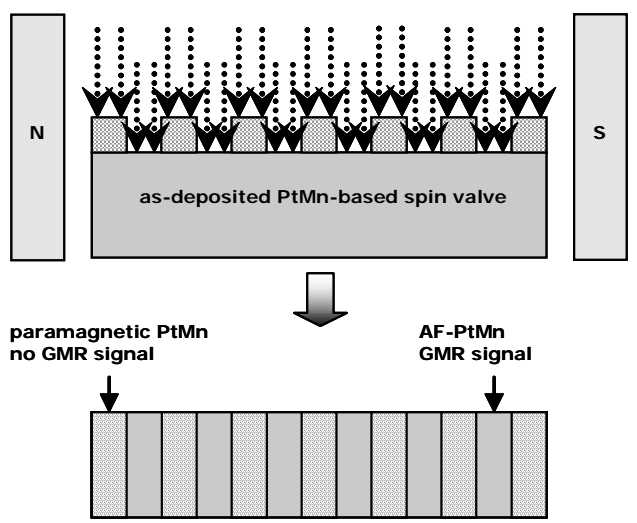

(b) $42 \mathrm{KeV}$ 0-ion irradiation

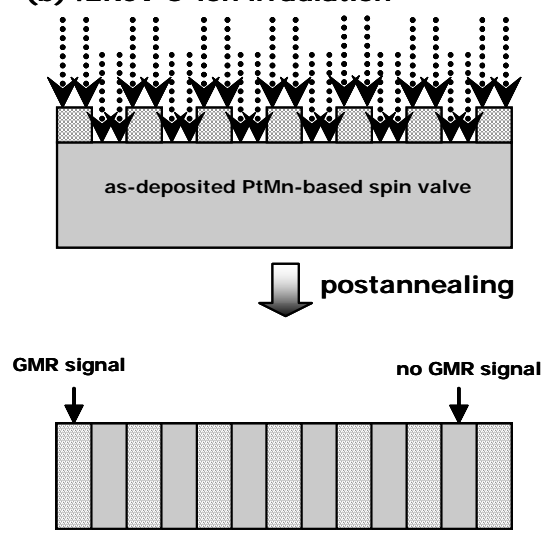

Figure 9. Schematic diagrams of magnetic patterning for PtMn-based spin valves by using (a) $2 \mathrm{MeV}$ He-ion irradiation and (b) $42 \mathrm{keV} \mathrm{O}$-ion irradiation. The areas exposed to He ions in (a) show a high exchange field and GMR. The areas exposed to O-ion irradiation show no GMR.

We first irradiate O-ions into the uncovered area, then the whole wafer is annealed at temperatures higher than $260{ }^{\circ} \mathrm{C}$ to achieve ordered PtMn. The area exposed to O-ion irradiation still have no GMR signal after annealing, but the area without O-ion irradiation shows a high GMR. Furthermore, the resistance of the irradiated area significantly increased by a factor of approximately 100, indicating that O-ion irradiation oxidized the layer and eliminated GMR. Although the resistance of the irradiated area significantly increased, current shunting still existed. Other ion sources or higher O-ion fluence may be needed to further increase the resistance of irradiated area and to suppress current flow in the irradiated area. Combining conventional photolithography [8] or non-contact stencil masks [16] with ion irradiation, GMR films can exhibit a high MR ratio in the area with He-ion irradiation, and a zero MR ratio with a high resistance in the area with O-ion irradiation; therefore, the dimension of currentperpendicular-to-plane (CPP) GMR cells may be magnetically patterned by using ion irradiation.

\section{CONCLUSION}

We have demonstrated that the highly ordered FePt $\mathrm{L}_{0}$ phase can be obtained by using an irradiation-induced heating process. He-ion irradiation introduces (1) microscopic energy transfer between incident ions and FePt atoms, (2) excess point defects in FePt films and (3) direct beam heating. Combining these effects, the ordering can be achieved at a nominal surface temperature as low as $230{ }^{\circ} \mathrm{C}$. In addition, we demonstrate that ion irradiation can be an alternative technique to fabricate patterned media.

We also showed that He-ion irradiation induced the direct-ordering of PtMn from fcc to the 
$\mathrm{L1}_{0}$ phase without post-annealing. An exchange field of 650 Oe and an MR ratio of $11 \%$ were obtained after He-ion irradiation. O-ion irradiation can completely destroy GMR by effectively oxidizing layers. Combining $\mathrm{He}$ and $\mathrm{O}$ ion-irradiation provides magnetic patterning for PtMn-based spin valves.

\section{ACKNOWLEDGMENT}

This work was supported in part by the National Science Council of R.O.C. under Grant number NSC 93-2112-M-007-033, Ministry of Economic Affairs of R.O.C. under Grants 94-EC-17-A-08-S1-0006, by NSF MRSEC DMR-0213808, Army Research Office DAAD 19-03-1-0298, DOE, INSIC and the W. M. Keck Foundation.

\section{REFERENCE}

1. A. P. Malozemoff, Phys. Rev. B 35, 3679 (1987).

2. D. Mauri, H. C. Siegmann, P. S. Bagus, and E. Kay, J. Appl. Phys. 62, 3047 (1987).

3. K. Coffey, M. A. Parker, and J. K. Howard, IEEE Trans. Magn. 31, 2737 (1995).

4. G. S. Chang, T. A. Callcott, G. P. Zhang, G. T. Woods, S. H. Kim, S. W. Shin, K. Jeong, C. N. Whang, and A. Moewes, Appl. Phys. Lett. 81, 3016 (2002).

5. D. M. Kelly, I. K. Schuller, V. Korenivski, K. V. Rao, K. K. Larsen, J. Bottiger, E. M. Gyorgy, and R. B. van Dover, Phys. Rev. B 50, 3481 (1994).

6. G. S. Chang, Y. P. Lee, J.Y. Rhee, J. Lee, K. Jeong, and C. N. Whang, Phys. Rev. Lett. 87, 067208 (2001).

7. D. Ravelosona, C. Chappert, V. Mathet, and H. Bernas, Appl. Phys. Lett. 76, 236 (2000).

8. L. Folks, J. E. E. Baglin, A. J. Kellock, M. J. Carey, B. D. Terris, and B. Gurney, IEEE Trans. Magn. 37, 1730 (2001).

9. A. Mougin, T. Mewes, M. Jung, D. Engel, A. Ehresmann, H. Schmoranzer, J. Fassbender, and B. Hillebrands, Phys. Rev. B 63, 060409(R) (2001).

10. C. H. Lai, C.H. Yang, R. T. Huang, C. W. Chen, F. R. Chen, J. J. Kai, H. Niu, J. Magn. Magn. Mater. 239, 390 (2002)

11. C. H. Yang, C. H. Lai, S. Mao, J. Appl. Phys. 93, 6596 (2003)

12. J. F. Ziegler, and J. P. Biersak, http://www.srim.org/.

13. B. E. Warren, X-Ray Diffraction (Addison-Wesley, Reading, MA, 1969) pp.208.

14. F. Seitz and J. S. Keohler, Solid State Phys. 2, 305 (1956).

15. D. H. Zhu, H. B. Lu, K. Tao, and B. X. Liu, J. Phys.: Condens. Matter 5, 5505 (1993).

16. B. D Terris, L. Folks, D. Weller, Appl. Phys. Lett. 75, 403 (1999) 